\title{
Testing configurations of Attractive Toxic Sugar Bait (ATSB) stations in Mali, West Africa, for improving the control of malaria parasite transmission by vector mosquitoes and minimizing their effect on non-target insects
}

Rabiatou A. Diarra

University of Science Techniques and Technology

Mohamed M. Traore

University of Science Techniques and Technology

Amy M Junnila

University of Science Techniques and Technology

Sekou F. Traore

University of Sciences Techniques and Technology

Seydou Doumbia

University of Science Techniques and Technology

\section{Edita E. Revay}

University of Science Techniques and Technology

Vasiliy D. Kravchenko

Tel Aviv University Department of Zoology

\section{Yosef Schlein}

Hebrew University Hadassah Medical School

Kristopher L. Arheart

University of Miami School of Medicine

Petranyi S. Gergley

University of Science Techniques and Technology

Axel Hausmann

Zoologische Staatssammlung Munchen

\section{Robert Beck}

Zoologische Staatssammlung Munchen

\section{Rui-de Xue}

Anastasia Mosquito Control District

\section{Alex M. Prozorv}

University of Sciences Techniques and Technology 


\section{Aboubakr S. Kone}

University of Science Techniques and Technology

\section{Silas Majambere}

University of Science Techniques and Technology

John Vontas

Foundation of Research and Technology Hellas Library

John C. Beier

University of Miami School of Medicine

Gunter Muller ( $\nabla$ guntercmuller@hotmail.com )

Hebrew University Hadassah Medical School https://orcid.org/0000-0002-7024-0179

\section{Research}

Keywords: Non-target organisms (NTOs), Anopheles gambiae s.I., ATSB, ASB, Diptera, Lepidoptera, Hymenoptera

Posted Date: January 22nd, 2021

DOI: https://doi.org/10.21203/rs.3.rs-46985/v2

License: (c) (i) This work is licensed under a Creative Commons Attribution 4.0 International License. Read Full License

Version of Record: A version of this preprint was published at Malaria Journal on April 14th, 2021. See the published version at https://doi.org/10.1186/s12936-021-03704-3. 


\section{Abstract}

Background Attractive Toxic Sugar Baits (ATSBs) successfully reduced Anopheles mosquito vector populations and malaria parasite transmission in Mali, but application methods need to be improved for wide-scale use, and effects on non-target organisms (NTOs) must be assessed. The goals of this study were to determine on a village level the effect of different outdoor configurations of ATSB bait stations to 1) achieve $>25 \%$ Anopheles mosquito vector daily feeding rate for both males and females and 2) minimize the effect on non-target organisms.

Methods Dye was added to Attractive Sugar Bait Stations (ASB - without toxin) to mark mosquitoes feeding on the sugar baits, and CDC UV light traps were used to monitor mosquitoes for the presence of the dye. Yellow plates, pitfall traps, Malaise traps, UV light traps, UV tray traps, and sweep nets were used to trap and sample non-target organisms (NTOs) for dye, indicating feeding on the ASB. ASB stations were hung on outer walls of village homes to determine the impact of different densities of ASBs $(1,2$, or 3 per home) as well as the impact of ASB height ( $1 \mathrm{~m}$ or $1.8 \mathrm{~m}$ above the ground on sugar feeding by anophelines. These experiments were carried out separately, on consecutive nights for mosquito and NTO monitoring. Eight villages in the Koulikoro province were chosen as the experimental locations.

Results The use of one ASB station per house marked $23.11 \%$ of female and $7.11 \%$ of male An. gambiae s.l. While two and three ASB stations per house gave feeding rates above the $25 \%$ goal, there was no statistical difference in the percentage of marked mosquitoes ( $p=0.3141$ females; $p=0.9336$ males). There was no difference in sugar feeding on ASB stations when hung at 1.0 and $1.8 \mathrm{~m}$ and $(\mathrm{p}=0.5170$ females; $p=0.9934$ males); however, ASBs at $1.8 \mathrm{~m}$ had less accidental damage from village residents and animals, and subsequent invasion of non-targets through rips or holes produced. ASB stations at 1.8 $\mathrm{m}$ above ground were fed on by three of seven monitored insect orders. Feeding rates were less than $0.015 \%$ of total trap catches and as low as $0.0001 \%$. The monitored orders were: Hymenoptera [ants (Formicidae), bees (Apidae), and wasps (Vespidae)], Lepidoptera (Rhopalocera, Bombyces, Geometroidea, Noctuoidea, Sphingidae, Pyraloidea), Coleoptera (Carabidae, Tenebrionidae, Scarabaeidae, Cerambycidae, and Chrysomelidae), Diptera (Brachycera, Chironomidae), Hemiptera (Cicadomorpha and Heteroptera), Neuroptera (Myrmeleontiformia) and Orthoptera (Caelifera and Ensifera). Using one or two stations limited evidence of NTO feeding to ants (Hymenoptera), Brachycera, Heteroptera, Noctuiodea, Rhopalocera, wasps (Vespidae) and wild bees (Apidae) (both Hymenoptera) and had a significantly reduced percentage of stained individuals compared to three stations which had the highest feeding rates amongst NTOs. The percentages of stained individuals were as follows: $6.84 \pm 2.03 \%$ Brachycera were stained followed by wasps (Hymenoptera: Vespidae) $5.32 \pm 2.27 \%$, and Rhopalocera $2.22 \pm 1.79 \%$. Hanging the optimal number of stations per house for catching mosquitoes (two) $1.8 \mathrm{~m}$ above ground, limited the groups of non-targets to Brachycera, Chironomidae, Noctuoidea, Rhopalocera, parasitic wasps and wasps (both Hymenoptera: Vespidae). The three most commonly stained non-target insect groups at this height were wasps (Vespidae) $(1.65 \pm 0.75 \%)$, Chironomidae $(0.99 \pm 0.37)$, and Brachycera (1.55 \pm $0.69 \%)$. Feeding at this height only occurred when stations were damaged. 
Conclusions The goal of marking one quarter of the total Anopheles mosquito vector population per day was obtained using 2 bait stations at $1.8 \mathrm{~m}$ height above the ground on the outer walls of houses. This configuration of ATSB stations also had minimal effects on non-target insects: only $0.0001 \%$ to $0.013 \%$ of specimens (in three orders) were marked. Stations hung $1.8 \mathrm{~m}$ above the ground had less accidental damage from passing people and livestock. The minimal marking of non-target insects may be attributed to visual orientation of non-mosquito insects while mosquitoes, are mostly guided by olfactory cues. Furthermore, the bait stations have a membrane cover, which if intact, is impenetrable to most sugar feeding non-target insects but is pierced by the stylets of the mosquito proboscis. Thus, most non-target insects are not exposed to the toxin even if they approach the bait stations.

\section{Introduction}

The search for methods to enhance the present arsenal of mosquito control continues as malaria remains a devastating disease [1]. A new method, Attractive Toxic Sugar Baits (ATSB), has been successfully used against populations of Anopheles as well as several other mosquito species [2,3,5-7]. Sugars are a staple diet of mosquitoes and the ATSB imitates the mosquito attraction to natural sources by olfactory cues and offers food that can be "spiked" with various toxins such as carbamates, pyrethroids, neonicotinoids, spinosyns, borates, and biopesticides [2-7]. Toxic sugar baits have been used as a foliar spray on green vegetation and blossoms [2-3] and also as an ATSB which can be hung on the external walls of houses or inside cisterns or drains [4].

The success of ATSB spray against mosquitoes, particularly on flowering vegetation [2], raised concerns as to whether ATSB, could affect populations of non-target organisms such as bees and butterflies. It was found that an unacceptable variety of species were stained when non-toxic Attractive Sugar Bait (ASB) was used to dye-mark non-target insects in simulated kill experiments [8,9]. The solution to spray only green vegetation with ATSB $[8,9,10]$ and to issue instructions with commercial ATSB formulations to only spray up to $5 \%$ of the green vegetation. The same concern with early bait station prototypes led to the suggestion that a physical barrier, such as a fine wire mesh cage, be added to the outside to prevent entry of the larger non-targets [8].

In this study, a new prototype ATSB station suitable for wide-scale use was tested and the goals were 1) to identify effective outdoor village-level configurations of bait stations needed to achieve a minimum average dye-marked vector prevalence rate of over $25 \%$ for both males and females, and 2) to determine bait station configurations that minimize the impact on non-target organisms (NTOs). The durability and efficacy of the bait over time was also evaluated.

\section{Materials And Methods}

\section{Villages}


Experiments were carried out in the following villages, located in the Koulikoro Province of Mali, West Africa: Tiko (12.13444, -8.396860), Balala (11.96599, -8.468310), Niaganabougou (12.15466, -8.308260), Sambadani (12.14454, -8.316880), Korea (12.04576, -8.399230), Krekrelo (11.98836, -8.551460), Cissebougou (12.09628, -8.372850), and Trekorou (12.068577, -8.314414). The villages, accessible by car, were within $10 \mathrm{~km}$ of the Niger River, and had high densities of An. gambiaes.l.

\section{Bait station construction and ASB Composition}

ASB consisted of the following: 22\% (w/w) date syrup as the attractant component, $77 \%(\mathrm{w} / \mathrm{w})$ brown sugar ("Nature Sugar" brown, Louis Dreyfus, Israel) as the feeding stimulus, 10\% (w/w) of a proprietary mixture of slow-release substances, (BaitStab ${ }^{\mathrm{T}}{ }^{\mathrm{M}}$, Westham Innovations Ltd., Tel Aviv, Israel) which is a proprietary preservative component, and $0.5 \%(\mathrm{w} / \mathrm{w})$ orange food dye (Carmoisine E122, Stern, Natanya, Israel). A similarly prepared solution with green food dye (Tartrazine 19140, Stern, Natanya, Israel) instead of orange was used for the $1.0 \mathrm{~m}$ height experiment. Bait stations were constructed using a white, rectangular plastic frame, $24 \mathrm{~cm}$ w by $36 \mathrm{~cm}$ l, with the ASB inside a proprietary, permeable, black SEBS (Styrene Ethylene Butylene Styrene) membrane (100 g of ASB inside 16 cells of membrane Westham Innovations LTD, Tel Aviv, Israel).

\section{Optimal bait station number on external house walls}

Monitoring in each of eight villages was carried out with ten CDC UV (John W. Hock Co., Gainesville, FL, USA) light traps, set in the center of the village, $5 \mathrm{~m}$ from houses and at least $10 \mathrm{~m}$ away from each other in a rough grid pattern for eighteen nights in 2016. ASB station coverage consisted of one, two or three bait station(s) with dye on every house in the village; if more than one bait station was used, they were situated on opposite walls at a height of $1.8 \mathrm{~m}$ (Supplemental Fig. 1). On August 17, 24, 30, a single ASB station was hung at $1.8 \mathrm{~m}$ above the ground on each house as well as on September 04, 10, 16, and 27. Two bait stations were hung per house on August 19 and 26, and on September 02, 05, 12, and 20. three bait stations were hung per house on August 21 and 29 as well as on September 09, 13 and 25. CDC Traps were set at 18:00 $\mathrm{h}$ and emptied at 06:00 $\mathrm{h}$ the following day.

\section{Effect of bait station height.}

Monitoring with CDC Traps from 18:00 $\mathrm{h}$ to $06: 00 \mathrm{~h}$ as described above was carried out on nine nights: July 25, 27, 29, August 05, 09, 08, 11, 12, and 15 with ASBs deployed in which 2 ASBs were situated on the same wall at $1.0 \mathrm{~m}$ and $1.8 \mathrm{~m}$ above the ground on two opposing walls of each house in the village. ASBs at $1.0 \mathrm{~m}$ contained green stain while the stain in the ASB positioned $1.8 \mathrm{~m}$ was orange.

\section{Performance of fresh versus aged baits.}

ASB coverage consisted of 2 bait stations on every house, side by side, $2 \mathrm{~m}$ apart, both $1.8 \mathrm{~m}$ above the ground, one fresh, the other aged under field conditions (by hanging outside near the study area) for 6 months each with differently coloured bait (orange and green). The trials ran for six days and nights, during which feeding rates of An. gambiae s.l. were evaluated and dates included October 1, 2, 6, 8, 11 
and 13. Mosquitoes were collected in each village with $10 \mathrm{CDC}$-UV light traps set in the center of the village, $5 \mathrm{~m}$ from houses but at least $10 \mathrm{~m}$ away from each other. Traps were set at 18:00 $\mathrm{h}$ and emptied at $06: 00 \mathrm{~h}$ the following day.

\section{NTO monitoring}

NTOs were monitored after the ASB deployment in each village with 50 yellow plates (yellow disposable plastic plates $25-\mathrm{cm}$ diameter filled with water and a drop of Triton X-100 as detergent), four Malaise traps (6 m; John W. Hock Co., Gainesville, FL, USA), two ultraviolet light traps (generator powered 250W ML light bulb mounted in front of a white $2 \mathrm{~m} \times 5 \mathrm{~m}$ white linen sheet), six ultraviolet tray traps [13], 50 pitfall traps (500 ml plastic cups buried to the rim in the ground, baited with $10 \mathrm{ml}$ vinegar), sweep nets (BioQuip, Rancho Dominguez, CA, USA) (operated by two collectors), and aerial hand nets (BioQuip, Rancho Dominguez, CA, USA). Collected insects were stored in paper envelopes and petri dishes at $-20^{\circ} \mathrm{C}$ before being processed for identification.

Stained specimens were separated from unstained specimens in the catches, stained specimens were pooled and identified with assistance from experts from the Entomological Department at the ZSM Natural History Museum (Zoologische Staatssammlung München). ASB feeding evaluation focused on seven of the most common orders. Feeding was determined by dissecting and examining guts for food dye under a 10X dissecting microscope. The insect orders were: Hymenoptera [ants (Formicidae) bees (Apidae), and wasps (Vespidae)], Lepidoptera (Rhopalocera, Bombyces, Geometroidea, Noctuoidea, Sphingidae, Pyraloidea), Coleoptera (Carabidae, Tenebrionidae, Scarabaeidae, Cerambycidae, and Chrysomelidae), Diptera (Brachycera, Chironomidae), Hemiptera (Cicadomorpha and Heteroptera), Neuroptera (Myrmeleontiformia) and Orthoptera (Caelifera and Ensifera).

\section{Statistical Methods.}

Bait Station Number Analysis. We used a generalized linear mixed model to compare the effect of the number of bait stations on the number of mosquitoes that fed on the bait and were caught in the traps. Female and male mosquitos were analyzed separately. The fixed effects in the model were village and number of bait stations which was a repeated measure. The random term was traps nested within villages that formed the error term for the repeated measure of number of bait stations. A compound symmetric covariance matrix was used to represent the correlated data structure. We included the total number of males and females trapped as an offset to produce model mean percent, standard error, and $95 \%$ confidence bounds. We also present $p$-values for comparisons of the mean percent of mosquitoes that fed on the bait among the number of bait stations.

Bait Station Height Analysis. This analysis compared the number of mosquitoes that fed on the bait and were caught by traps at a height of $1.0 \mathrm{~m}$ and $1.8 \mathrm{~m}$. We used a generalized linear mixed model for Poisson outcome: the number of mosquitoes caught. Female and male mosquitos were analyzed separately. The fixed effects in the model were village and trap height which was a repeated measure. The random term was traps nested within villages that formed the error term for the repeated measure of 
height. A compound symmetric covariance matrix was used to represent the correlated data structure. We used the total number of males and females trapped as an offset to produce model mean percent, standard error, and $95 \%$ confidence bounds. We also present p-values for the comparison of the mean percent of mosquitoes that fed on the bait at each trap height.

Aged versus fresh baits. We used a generalized linear mixed model to compare the effect of bait age on the relative number of mosquitoes that fed on fresh and 6-month old ASB stations. Female and male mosquitoes were analyzed separately. The fixed effects in the model were village and bait age which was a repeated measure. The random term was traps nested within villages that formed the error term for the repeated measure of number of bait stations. A compound symmetric covariance matrix was used to represent the correlated data structure. We included the total number of males and females trapped as an offset to produce model mean percent, standard error, and 95\% confidence intervals. We also present pvalues for comparisons of the mean percent of mosquitoes that fed on the bait between the two ages of the bait.

Effect of bait station number on non-targets: We used a generalized linear mixed model to compare the effect of the number of bait stations on the number of insects that fed on the bait and were caught in the traps. The fixed effects in the model were village, type of insect, number of bait stations, and the interaction of insect type with number of bait stations. Type of insect and number of bait stations were repeated measures. The random term was traps nested within villages that formed the error term for the repeated measures. A compound symmetric covariance matrix was used to represent the correlated data structure. We included the total number of insects trapped as an offset to produce model mean percent, standard error, and 95\% confidence bounds. For each insect group, we also present p-values for comparisons of the mean percent of insects that had fed on the bait, between number of bait stations.

Effect of height on non-targets: This analysis compared the number of insects that fed on the bait at a height of $1.0 \mathrm{~m}$ and $1.8 \mathrm{~m}$. Separate analyses were performed for the different heights because the type of insects caught were different, depending on the height of the bait station. We used a generalized linear mixed model for Poisson outcome: the number of insects that fed on the bait. The fixed effects in the model were village and type of insect which was a repeated measure. The random term was traps nested within villages that formed the error term for the repeated measure of type of insect. A compound symmetric covariance matrix was used to represent the correlated data structure. We included the total number of insects trapped as an offset to produce model mean percent, standard error, and 95\% confidence bounds. We also present $p$-value for the comparison among the mean percent of each type of insect that had fed on the bait.

Analyses were carried out using SAS 9.4 (SAS Institute, Inc.; Cary, NC). The two-tailed alpha level was used to determine the statistical significance of all statistical tests.

\section{Results}

Choice of bait station number on the external walls of huts. 
In the seven trials with a single bait station $23.1 \%$ of the females and $14.6 \%$ of the males were dye marked by ASB. During the six trials using two bait stations per building, the percentage of dye marked females rose to $33.75 \%$ of the females and $30.62 \%$ of the males. Three bait stations increased the percentage of the labeled Anopheles gambiae s.I. only slightly to $39.11 \%$ females (330/ 826 ) and $32.77 \%$ males.

Using one station did not achieve male or female average daily vector feeding rates of $25 \%$ (Table $1 \mathrm{~A}$ ). Though there was a significant difference in labeled males and females between two and three bait stations, the differences only amounted to 2.15 and $5.36 \%$ respectively. Therefore, in subsequent experiments, it was determined that two bait stations hung from each building was sufficient to achieve target daily feeding rate of $25 \%$.

\section{Choice of bait station height on the external walls of huts.}

During nine trials, a total of 1,114 female and 665 male Anopheles gambiae s.l. were collected. These included $14.22 \%$ of the females and $12.06 \%$ of the males that fed from the stations $1.8 \mathrm{~m}$ above the ground, and $11.86 \%$ of the females and $12.20 \%$ of the males that fed on the stations $1.0 \mathrm{~m}$ above the ground (Figure 2; Table 2). There was no statistical difference between the number of males versus females stained by bait stations hung at $1.0 \mathrm{~m} \mathrm{(} \mathrm{p}=0.3755 ; 95 \% \mathrm{Cl} 0.00$ to 11.96$)$ or at $1.8 \mathrm{~m}(\mathrm{p}=0.518$ $95 \% \mathrm{Cl} 0.2605$ to 15.74$)$. There was also no difference between females labeled at $1.0 \mathrm{~m}$ versus those labeled at $1.8 \mathrm{~m} \mathrm{(} \mathrm{p}=0.5170 ; 95 \% \mathrm{Cl} 0.00$ to 11.18$)$ or males labeled at $1.0 \mathrm{~m}$ versus labeling at a height of $1.8 \mathrm{~m} \mathrm{(} \mathrm{p}=0.9934 ; 95 \% \mathrm{Cl}-8.08$ to 7.40$)$. The remainder of the caught mosquitoes (50.34\%) were not labeled.

Thirty-four (out of 1,409) of the lower stations were damaged accidentally by humans and animals walking by, resulting in insect invasion (which means insects found inside of a bait station). Most observed insect invasions (29 out of 34) were by ants, usually on one of the damaged stations. Social wasps invaded one of the higher stations while bees invaded one of the lower damaged stations.

The mean difference in the numbers of marked An. gambiae s.l. that had been labeled by ASB at a height of $1.8 \mathrm{~m}$ versus $1.0 \mathrm{~m}$ were not significantly different for either females $(P=0.5170)$ or males $(P=0.9934)$.

\section{Performance of fresh versus aged baits.}

In the experiment comparing fresh and aged ASB bait stations, 796 female and 251 male Anopheles were collected. From those, $16.66 . \%$ and $16.19 \%$ of the females fed from fresh and aged bait stations, respectively. Of the males, $15.28 \%$ and $15.12 \%$ fed on fresh vs aged bait respectively. There were no statistical differences between males and females feeding on fresh vs. aged baits (Table 3). 
The mean difference in the numbers of marked An. gambiae s.l. that had been labeled by ASB when fresh and aged bait stations were used was not significant for females $(P=0.4239)$ or males $(P=0.8158)$

\section{Correlation between the number of ASB stations and the presence of the dye marker in non-target insect species.}

To determine the impact of number of stations on non-targets insects, 57,902 captured insects from all villages were pooled and dissected. The dye marker was observed in $162(0.003 \%)$ specimens belonging to different insect groups as shown in Table 4.

The combined catches caught a wide variety of stained insects; 22 groups belonging to seven orders were stained (Table 4). The highest number of stained specimens were from Brachycera (5.73 $\pm 1.61 \mathrm{SE})$, Vespidae (4.91 $\pm 1.33 \mathrm{SE})$, and Myrmeleontiformia (3.02 $\pm 1.59 \mathrm{SE})$. Except for Heteroptera, the number of dye-marked specimens was in proportion to the number of bait stations hung (Table 5 ) as determined by the average catches of the various NTO trapping methods.

\section{Relationship between height of placement of ASB stations and the presence of dye marker in non-target insect species.}

The availability of bait stations at a height of $1.0 \mathrm{~m}$ and $1.8 \mathrm{~m}$ to non-target insects was compared. A total number of 34,401 specimens was captured and dissected, 446 of which had dye labeled by the color of low hanging stations, and 32 specimens were marked by the dye that was within the high hanging stations (Table 6$)$. In total, $0.0131 \%$ of the collected specimens were dye labeled $(0.013 \%$ from exposure to ASBs hung at $1.0 \mathrm{~m}$ and $0.0001 \%$ of specimens were exposed to dye at ASB stations hung at $1.8 \mathrm{~m}$ ).

With bait stations hung on walls at $1.8 \mathrm{~m}$ versus $1.0 \mathrm{~m}$, non-targets stained were lower by $91.93 \%$. Brachycera, wasps (Vespidae), and Chironomidae were predominant in the dye-marked group and had significantly higher mean numbers compared to other insects (Table7).

\section{Discussion}

The potential of Attractive Toxic Sugar Baits (ATSB) for control has been shown against populations of Anopheles vectors of malaria as well as several other mosquito species $[2,3,5-7,14,15,16]$. This study investigated ways to achieve adequate feeding by anophelines while minimizing exposure of the toxic bait to non-target organisms and using minimal investment of materials.

The ATSB system has several advantages. First, the proprietary SEBS membrane in this model bait station is easily penetrable by mosquito mouth parts so that they can land, probe and feed 
(Supplementary figure A). Most insects that ingest sugars from exposed sources such as floral honeydew lack appropriate mouth parts to penetrate the membrane and feed. Second, because it can be used with several classes of insecticide [4-6] it can help alleviate the problem of resistance, and the flexibility of ATSB also allows use of low toxicity or arthropod-specific insecticides [2]. Last, it can be used as a spray or as a bait station depending on the needs of the user. The baits are used outdoors where the problem of reducing mosquitoes lies, in contrast to bed nets and indoor residual spraying. Finally, ATSB has inherently low primary and secondary toxicity towards non-target organisms $[6,8-10]$ and its effect is limited to sugar feeding insects that respond to the particular attractant of the baits. In previous studies, when sprayed on flowering vegetation where the local flora also exerts its attraction, aside from mosquitoes ATSB, without toxin, was fed upon by Diptera and generally it dye-marked up to $18 \%$ of the non-target insects on a daily basis [8,11]. Dye-labelled Hymenoptera represented $15 \%$ of the caught population samples, Lepidoptera were $7 \%$, and the remaining orders accounted for $5 \% \quad 1.5 \%$. [8].

The advance from foliar ATSB spray to bait stations further reduced the number of non-target insects that fed on the bait [9], perhaps because bait stations, along with green vegetation, are not optical targets like flowers for sugar feeders while night active mosquitoes are drawn to the attractive scent [9]. In the current study, it was discovered that optimization of bait station placement could reduce the number of dye-marked specimens of non-target insect groups (Tables 5 and 6 ) for two reasons: 1 ) such locations were less in the course of their random flight paths and 2) stations could be moved out of the way of potential damage. Reducing the number of damaged stations could decrease feeding by non-target organisms. Feeding by mosquitoes was not affected by height but feeding by NTOs was higher among ATSBs at the lower height and therefore, the study indicates that bait stations should be placed higher on the walls of house (1.8m; Table $2 \mathrm{~A})$. Presentation of two bait stations with ASB marker per house led to a higher proportion of dye- marked mosquitoes than the presentation of one station. Placing three bait stations per house did not significantly increase the number of dye marked mosquitoes but it had the drawback of increasing the proportion of dye marked non-target insects (Table 1, Table 5) as well as requiring more labour to hang and requiring more expenses. Regardless of the number of stations used, the number of stained non-targets was low; 57,902 non-target insect specimens were caught during the "number of stations" experiments, only 162 of which $(0.0003 \%)$ were dye-marked.

Even though height affects the number of stained non-targets, those numbers are ultimately very low. A total of 34,401 stained non-targets were captured, $446(0.013 \%)$ had the dye of the lower stations while $32(0.0001 \%)$ had the dye of the higher stations. When bait stations were hung at $1.0 \mathrm{~m}$, the majority of marked specimens in descending order were Brachycera (midges and other small flies), wasps (Vespidae), and honey bees (Apidae). At this height, day-to-day activities of residents caused minor damage that exposed the bait and allowed feeding from the stations by non-targeted organisms. This damage appears to be the major reason since the labelling of insects was diminished by about $90 \%$ when the ASB marker stations were at a height of $1.8 \mathrm{~m}$. It is specifically noteworthy that honeybees were not marked by ASB stations at a height of $1.8 \mathrm{~m}$ (Tables 5 and 6 ). 
The current study as with earlier observations $[8-10,14]$ shows that except for mosquitoes ATSB mainly affect Diptera (Brachycera), and to a lesser extent Hymenoptera (wasps, ants and honey bees) unless properly deployed. Much of the affected Diptera were filth flies that often rest on the walls of houses (observations of the authors). Altogether, the study shows that in these Malian villages ATSB stations placed at a height of $1.8 \mathrm{~m}$ maintain their effect on mosquitoes, and unlike lower bait station positions, have a negligible effect of non-target insects.

Optimization of ATSB deployment, either as a barrier spray [12] on green vegetation or even more so as bait stations, can further increase the specificity of ATSB and also increase the kill rate on mosquitoes. We now have evidence that the problem of affecting non-target insects can be minimised through optimising deployment. These principles of establishing efficient use and minimising NTO impact are simple enough to be transferable throughout Africa and globally though further testing in a number of different host countries would be needed to demonstrate this. Improvement of the protective membrane to resist damage and leakage will also most likely further reduce non-target impact. This demonstration in Mali of how bait station placement and configuration can minimize effects of ATSB on non-target insects provides a strong supportive case that ATSB stations can be used with confidence for the control of malaria parasite transmission by Anopheles mosquitoes with minimal harmful environmental impacts.

\section{Declarations}

\section{Acknowledgements}

The authors would like to thank the community members in the villages in Mali for their participation. This study was supported by the Innovative Vector Control Consortium. This project was also supported by NIH grants U19AI129387 and R01Al100968. The funders had no role in study design, data collection and analysis, decision to publish, or preparation of the manuscript. The content is solely the responsibility of the authors and does not necessarily represent the official views of the National Institutes of Health. The authors declare that no competing interests exist in relation to this project.

\section{Author Contributions}

GCM YS RAD and JCB conceived and designed the study. GCM, RAD, AMP, ASK, MMT, SFT, JV, AH, RB, PG, VDK, SD, and EER supervised and carried out the field work. AJ drafted the manuscript. SM and RX assisted in drafting the manuscript. KLA carried out statistical analyses.

\section{Ethics approval and consent to participate}

All the work involving the use of human volunteers in experimental homes and HLCs was approved by the ethical review committee of the University of Sciences Techniques and Technology (IRB 2015/107/CE/FMPOS).

\section{Consent for publication}


All authors contributed to the interpretation of the data and approved the final manuscript.

\section{Competing interests}

The authors declare that they have no competing interests.

\section{Availability of data and materials}

All data generated and analysed during this study are included in this published article.

\section{Funding}

This study was supported by the Innovative Vector Control Consortium. This project was also supported by NIH grants U19Al129387 and R01Al100968.The funders had no role in study design, data collection and analysis, decision to publish, or preparation of the manuscript. The content is solely the responsibility of the authors and does not necessarily represent the official views of the National Institutes of Health. The authors declare that no competing interests exist in relation to this project.

\section{References}

1. WHO. World Malaria report 2018. Geneva: World Health Organization; 2018.

2. Schlein Y, Müller GC. An approach to mosquito control: using the dominant attraction of flowering Tamarix jordanis trees against Culex pipiens. J Med Entomol. 2008;45:384-90.

3. Müller GC, Junnila A, Qualls W, Revay EE, Kline DL, Allan S, Schlein Y, Xue R. Control of Culex quinquefasciatus in a storm drain system in Florida using attractive toxic sugar baits. Med Vet Entomol. 2010;24:346-51.

4. Schlein Y, Müller GC. Diurnal resting behavior of adult Culex pipiens in an arid habitat in Israel and possible control measurements with toxic sugar baits. Acta Trop. 2012;124:48-53.

5. Junnila A, Revay EE, Müller GC, Kravchenko V, Qualls WA, Allen SA, Beier JC, Schlein Y. Efficacy of attractive toxic sugar baits (ATSB) against Aedes albopictus with garlic oil encapsulated in betacyclodextrin as the active ingredient. Acta Trop. 2015;152:195-200.

6. Revay EE, Schlein Y, Tsabari O, Kravchenko V, Qualls W, De-Xue R, Beier JC, Traore SF, Doumbia S, Hausmann A, Müller GC. Formulation of attractive toxic sugar bait (ATSB) with safe EPA-exempt substance significantly diminishes the Anopheles sergentii population in a desert oasis. Acta Trop. 2015;150:29-34.

7. Müller GC, Junnila A, Schlein Y. Effective control of adult Culex pipiens by spraying an attractive toxic sugar bait solution in the vegetation near larval habitats. J Med Entomol. 2010;47:63-6.

8. 8. Qualls WA, Müller GC, Revay EE, Allan SA, Arheart KL, Beier JC, Smith ML, Scott JM, Kravchenko VD, Hausmann A, Yefremova ZA. Evaluation of attractive toxic sugar bait (ATSB)-barrier for control of vector and nuisance mosquitoes and its effect on non-target organisms in sub-tropical environments in Florida. Acta Trop. 2014;131:104-10. 
9. Revay EE, Müller GC, Qualls WA, Kline DL, Naranjo DP, Arheart KL, Kravchenko VD, Yefremova Z, Hausmann A, Beier JC, Schlein Y. Control of Aedes albopictus with attractive toxic sugar baits (ATSB) and potential impact on non-target organisms in St. Augustine, Florida. Parasitol Res. 2014;113:73-9.

10. Khallaayoune K, Qualls WA, Revay EE, Allan SA, Arheart KL, Kravchenko VD, Xue RD, Schlein Y, Beier JC, Müller GC. Attractive toxic sugar baits: control of mosquitoes with the low-risk active ingredient dinotefuran and potential impacts on non target organisms in Morocco. Environ Entomol. 2013;42(5):1040-5.

11. Fiorenzano JM, Koehler PG, Xue RD. Attractive toxic sugar bait (ATSB) for control of mosquitoes and its impact on non-target organisms: a review. Int J Environ Res Public Health. 2017;14:398.

12. Qualls WA, Müller GC, Revay EE, Kravchenko VD, Yefremova ZA, Hausmann A, Smith ML, Beier JC, Xue RD. Large scale control of Anopheles crucians with attractive toxic sugar bait (ATSB) barrier treatment and effects on non-target organisms in St Augustine, Florida. $79^{\text {th }}$ Annual meeting of the American Mosquito Control Association, Abstract 225.

13. Qualls WA, Müller GC, Khallaayoune K, Revay EE, Zhioua E, Kravchenko VD, Arheart KL, Xue RD, Schlein Y, Hausmann A, Kline DL, Beier JC. Control of sand flies with attractive toxic sugar baits (ATSB) and potential impact on non-target organisms in Morocco. Parasites Vectors. 2015;8(1):87.

14. Müller GC, Beier JC, Traore SF, Toure MB, Traore MM, Bah S, Doumbia S, Schlein Y. Successful field trial of attractive toxic sugar bait (ATSB) plant-spraying methods against malaria vectors in the Anopheles gambiae complex in Mali, West Africa. Malaria J. 2010;9(1):210.

15. Beier JC, Müller GC, Gu W, Arheart KL, Schlein Y. Attractive toxic sugar bait (ATSB) methods decimate populations of Anopheles malaria vectors in arid environments regardless of the local availability of favoured sugar-source blossoms. Malaria J. 2012;11(1):1-7.

\section{Tables}

The Tables are provided in the Supplementary Files

\section{Supplementary Files}

This is a list of supplementary files associated with this preprint. Click to download.

- TablesAlleditableJan152021.xlsx 